\title{
Horner syndrome following thyroidectomy
}

\author{
Domenico Italiano $\cdot$ Simona Cammaroto $\cdot$ \\ Clemente Cedro · Placido Bramanti · \\ Edoardo Ferlazzo
}

Received: 15 January 2010/ Accepted: 2 November 2010/Published online: 19 November 2010

(C) Springer-Verlag 2010

Dear Editor,

Horner syndrome (HS) is due to the disruption of sympathetic innervation of the eye anywhere along its threeneuron circuit. The typical clinical feature includes a triad of ipsilateral blepharoptosis, pupillary miosis, and facial anhydrosis. Neoplasm of lung apex (Pancoast tumor), vascular injuries, syringohydromyelia, carotid dissection or fibromuscolar dysplasia, are the most common causes [1]. HS has only rarely been reported after thyroidectomy, with only 28 cases mostly published in surgical journals [2, 3].

Herein, we report a 56-year-old man exhibiting HS after total thyroidectomy. Patient came at our observation 40 days after surgical intervention for multinodular goiter. Histological examination on specimens from ablate thyroid tissue was negative for neoplasms. Immediately after awakening from anesthesia, mild myosis, eyelid ptosis and enophthalmos of the right eye were evident. Patient also exhibited a bitonal voice. Neurological examination was otherwise normal. Emiphacial anhydrosis was not noticed by patient. Laryngoscopy examination revealed right vocal cord paralysis. Brain MRI, chest X ray, single fiber EMG and neck CT scan were normal. At 2-month follow-up, a slight improvement of eyelid ptosis was observed.

The possible causes of HS following thyroidectomy include post-operative hematoma compressing the cervical

D. Italiano · S. Cammaroto · P. Bramanti - E. Ferlazzo $(\square)$

IRCCS Centro Neurolesi "Bonino-Pulejo",

Messina, Italy

e-mail: edoferl@hotmail.it

C. Cedro

Department of Neurosciences,

Psychiatric and Anaesthesiological Sciences,

University of Messina, Messina, Italy sympathetic chain, ischemia-induced neural damage caused by lateral ligature on inferior thyroid artery trunk, stretching of the cervical sympathetic chain by the retractor, damage to the anastomosis between the cervical sympathetic chain and the recurrent laryngeal nerve during its identification [2]. Prognosis is usually poor with $70 \%$ of patients presenting permanent damage or incomplete recovery. Complete remission may occur after long time (up to 15 months). In the reported case, post-operative hematoma or adherences were ruled out by neck CT scan. Since right vocal cord paralysis, probably related to the deficit of the inferior laryngeal nerve, was also present, we argue that mechanical damage on the anastomosis of the recurrent laryngeal nerve and sympathetic nervous branches around the inferior thyroid artery, might cause HS in our patient.

\section{References}

1. Reede DL, Garcon E, Smoker WR, Kardon R (2008) Horner's syndrome: clinical and radiographic evaluation. Neuroimaging Clin N Am 18:369-385

2. Cozzaglio L, Coladonato M, Doci R, Travaglini P, Vizzotto L, Osio M, Gennari L (2008) Horner's Syndrome as a complication of thyroidectomy: report of a case. Surg Today 38:1114-1116

3. Solomon P, Irish J, Gullane P (1993) Horner's syndrome following a thyroidectomy. Otolaryngology 22:454-456 